\title{
4-HPR impairs bladder cancer cell migration and invasion by interfering with the Wnt5a/JNK and Wnt5a/MMP-2 signaling pathways
}

\author{
YUANFEI CAO ${ }^{1,2^{*}}$, XIAOLONG WANG ${ }^{2 *}$, CHANG XU $^{2}$, ZHENGYAN GAO $^{2}$, \\ HAIHONG ZHOU ${ }^{2}$, YONGZHI WANG ${ }^{2}$, RUI CAO ${ }^{2}$, TAO LIU ${ }^{2}$ and TONGZU LIU ${ }^{2}$ \\ ${ }^{1}$ Department of Urology, Taizhou People's Hospital of Jiangsu Province, Taizhou, Jiangsu 225300; \\ ${ }^{2}$ Department of Urology, Zhongnan Hospital, Wuhan University, Wuhan, Hubei 430071, P.R. China
}

Received April 10, 2015; Accepted May 26, 2016

DOI: $10.3892 / \mathrm{ol} .2016 .4908$

\begin{abstract}
Inordertoidentify the anti-invasive and anti-metastatic effect of the synthetic retinoid N-(4-hydroxyphenyl) retinamide (4-HPR) on the human bladder cancer EJ cell line, and to study its impact on the expression of wingless-type mouse mammary tumor virus integration site family, member $5 \mathrm{a}$ (Wnt5a), the phosphorylation of c-Jun N-terminal kinase (JNK), the expression levels of matrix metalloproteinase-2 (MMP-2), and the migration and invasion of EJ cells, migration and Matrigel invasion assays, as well as western blot analyses, were used in the present study. The results of the migration and Matrigel invasion assays indicated that the inhibitor of JNK SP600125 could inhibit the effect of 4-HPR on EJ cells. The expression of Wnt5a and MMP-2, and the phosphorylation of JNK, were analyzed by western blotting. The data revealed that 4-HPR inhibited the migration and invasion of bladder cancer cells through stimulating Wnt5a activation, causing the downregulation of MMP-2 expression and enhancing the phosphorylation of JNK in these cells. However, JNK signaling did not appear to have a direct effect on the expression of MMP-2. The present study demonstrated that 4-HPR may be a potent anti-invasive and anti-metastatic agent that functions via the Wnt5a/JNK and Wnt5a/MMP-2 signaling pathways.
\end{abstract}

\section{Introduction}

Bladder cancer remains a common malignant disease in the USA, with an estimated incidence of $\sim 73,500$ cases in 2012 , and accounts for almost 15,000 mortalities per year (1).

Correspondence to: Professor Tongzu Liu, Department of Urology, Zhongnan Hospital, Wuhan University, 169 Donghu Road, Wuhan, Hubei 430071, P.R. China

E-mail: liutongzu@163.com

*Contributed equally

Key words: 4-HPR, Wnt5a, JNK, MMP-2, migration and invasion, bladder cancer
Approximately $75 \%$ of newly diagnosed urothelial bladder cancers (UBCs) are non-invasive, but have a high rate of recurrence and progression despite local therapy (2). This frequency, coupled with the relapsing nature of $\mathrm{UBC}$, means that $\mathrm{UBC}$ poses an enormous burden on healthcare systems $(2,3)$. Therefore, it is of enormous importance to identify novel effective therapeutics or chemoprevention to halt and delay the recurrence and progression of UBC.

$\mathrm{N}$-(4-hydroxyphenyl) retinamide (4-HPR) is one of the most studied retinoid analogs for use in cancer prevention (4-6). 4-HPR inhibits the growth of breast, prostate and ovarian cancers in animal models (4-6). Compared with other vitamin A analogues, 4-HPR-induced cell differentiation is weak, its cancer chemopreventive effect is remarkable and its anti-cancer activity is apparent (7). In addition, 4-HPR has exhibited potent preventive effects in a rodent mammary tumor model and less toxicity than retinoic acid, and has also been tested in a large breast cancer prevention trial (8). Therefore, 4-HPR may exert a chemo-preventive effect on UBC growth.

Matrix metalloproteinases (MMPs) are a super-family of proteolytic enzymes capable of degrading the extracellular matrix (ECM) and basement membrane (9). Tumor invasion, metastasis and angiogenesis require controlled degradation of the ECM, and increased expression of MMPs is associated with tumor invasion and metastasis of malignant tumors with different histogenetic origin $(10,11)$. Of all the MMPs described so far, MMP-2 (also known as gelatinase A or type IV collagenase) has been reported to be closely linked to cancer (12). The principal activity of MMP-2 is the hydrolysis of gelatin and type IV collagen, which are the main structural components of basement membranes (13). Furthermore, sulforaphane inhibits the migration of human bladder cancer cells via the cyclooxygenase-2/MMP-2 signaling pathway (14).

Wingless-type mouse mammary tumor virus integration site family (Wnt) proteins play essential roles in developmental and physiological processes by regulating various cell functions, including proliferation, differentiation, apoptosis, survival, adhesion, migration and polarity (15). Wnt5a is a member of the Wnt family of secreted glycoproteins, which are involved in tumor progression and malignant initiation (16). The non-canonical Wnt signaling consists of at least the Wnt/c-Jun 
$\mathrm{N}$-terminal kinase (JNK) and $\mathrm{Wnt} / \mathrm{Ca}^{2+}$ signaling pathways, which are considered to have crucial functions in the regulation of cell migration and polarity (17). Wnt5a is a representative Wnt protein that activates non-canonical Wnt signaling (17). It has been proposed that Wnt5a signaling is activated by the Wnt/JNK signaling pathway, which regulates convergent extension movements in Xenopus gastrulation, and/or inhibition of the $\beta$-catenin/transcription factor signaling pathway (18). JNK is known to play a role in cell migration by phosphorylation of paxillin Ser178, a focal adhesion molecule (19). In addition, Wnt5a signaling is correlated with infiltrative activity in human glioma by inducing cellular migration and MMP-2 expression (20). Therefore, the present authors hypothesized that 4-HPR inhibits migration and invasion of bladder cancer cells via Wnt5a/JNK or Wnt5a/MMP-2 signaling.

\section{Materials and methods}

Cell cultures. The EJ cell line was purchased from the Cancer Institute and Hospital of the Chinese Academy of Medical Sciences (Beijing, China), and cultured in RPMI-1640 medium (Gibco; Thermo Fisher Scientific, Inc., Waltham, MA, USA) supplemented with $10 \%$ foetal calf serum (Gibco; Thermo Fisher Scientific, Inc.). 4-HPR was purchased from Sigma-Aldrich (St. Louis, MO, USA), dissolved in dimethyl sulfoxide (DMSO) as $10-\mathrm{mM}$ stock solutions and stored in the dark at $-20^{\circ} \mathrm{C}$. Lipofectamine 2000 reagent was purchased from Invitrogen (Thermo Fisher Scientific, Inc.). The inhibitor of JNK SP600125 (Cayman Chemical Company, Ann Arbor, MI, USA) was applied to the culture medium at a final concentration of $20 \mu \mathrm{M}$.

Cell proliferation, apoptosis, chemotaxis and invasion assays. All proliferation and viability assays were based on the 3-(4,5-dimethylthiazol-2-yl)-2,5-diphenyltetrazolium bromide (MTT) method. Cells were seeded in a 96-well plate at a density of 2,000 cells/well. The cells were treated with $0,2.5$, 5 and $10 \mu \mathrm{M} 4-\mathrm{HPR}$, and allowed to grow for 24, 48, 72 and $96 \mathrm{~h}$. At the end of the experiment, the media were removed, and DMSO was added with MTT solubilization solution. Absorbance was measured at $595 \mathrm{~nm}$. Matrigel invasion assays were used to assess the effect of 4-HPR in EJ cells. The 8- $\mu \mathrm{m}$ pore size polycarbonate nucleopore filter inserts in a 24-well transwell chamber (BD Biosciences, Franklin Lakes, NJ, USA) were coated with $60 \mu \mathrm{g} /$ filter Matrigel (BD Biosciences). 4-HPR-treated EJ cells at a density of 40,000 cells/well were seeded into the upper part of the Matrigel-coated filter, and RPMI-1640 medium supplemented with $10 \%$ heat-inactivated fetal bovine serum (Gibco; Thermo Fisher Scientific, Inc.) was added to the lower part. After $48 \mathrm{~h}$, the cells that had migrated through the Matrigel and the $8-\mu \mathrm{m}$ pore-size membrane were fixed, stained and counted under a light microscope. Migration assays adopted the same method that invasion assays, with the exception of the use of uncoated filters, and were conducted for $24 \mathrm{~h}$.

Protein extraction and western blot analyses. Proteins were obtained from EJ cells after 48-h culture in the absence or presence of 4-HPR, or after $24 \mathrm{~h}$ in the presence of SP600125. The cells were then lysed in radioimmunoprecipitation assay buffer containing protease inhibitors. Protein concentration was determined with the DC Protein Assay kit (Bio-Rad Laboratories, Inc., Hercules, CA, USA). Equal amounts of samples were resolved by $10 \%$ sodium dodecyl sulfate-polyacrylamide gel electrophoresis, transferred to nitrocellulose membranes and probed at $4^{\circ} \mathrm{C}$ overnight with the following anti-human antibodies: Mouse monoclonal anti-Wnt5a antibody (ab130163; 1:1,000; Abcam, Cambridge, UK), rabbit monoclonal anti-MMP-2 (13132; 1:1,000; Cell Signaling Technology, Inc., Danvers, MA, USA), rabbit monoclonal anti-JNK (9252; 1:1,000; Cell Signaling Technology, Inc.) and rabbit monoclonal anti-phospho-JNK on Thr183/Tyr185 (4668; 1:1,000, Cell Signaling Technology, Inc.).

After washing, the blots were incubated for $1 \mathrm{~h}$ at room temperature with horseradish peroxidase (HRP)-conjugated secondary antibodies (RPN2232; GE Healthcare Life Sciences, Chalfont, UK), and specific complexes were revealed by enhanced chemiluminescence (GE Healthcare Life Sciences). An anti-glyceraldehyde 3-phosphate dehydrogenase antibody conjugated to HRP (Novus Biologicals, LLC, Littleton, CO, USA) was utilized as a loading control for all samples.

Gene knockdown. For gene knockdown, small interfering RNA (siRNA) duplexes (Shanghai GenePharma Co., Ltd., Shanghai, China) targeting Wnt5a (5'-GACCUGGUCUAC AUCGACCTT-3' and 5'-CCGCGAGCGGGAGCGCAT-3') and scrambled sequence Wnt5a siRNA (5'-GGUCCAGUC AGCCACUCUATT-3') were transfected into EJ cells by using Lipofectamine 2000 reagent. Knockdown efficiency was evaluated $48 \mathrm{~h}$ after transfection by measuring the protein levels in the cell lysates.

Statistical analysis. The normality and homogeneity of variance were tested prior to statistical analysis with Prism 5 using the Kolmogorov-Smirnov test. Data were expressed as the mean \pm standard deviation, unless otherwise noted. The differences between groups were analyzed using a two-tailed Student's $t$-test and the null hypothesis was rejected at the 0.05 level. For all statistical tests, two-tailed $\mathrm{P}<0.05$ was considered to indicate a statistically significant difference.

\section{Results}

4-HPR decreases cell proliferation, migration and invasion. The action of 4-HPR on bladder cancer cell proliferation was assessed by treating EJ cells with a range of 4-HPR concentrations. All the concentrations tested inhibited cell growth, with statistically significant differences only after 96-h exposure (Fig. 1A). Migration of cancer cells is one of the key factors responsible for cancer metastasis (10). To metastasize, cancer cells must migrate from the original growth site, invade surrounding tissues and locate to other parts of the body through the blood or the lymphatic system (10). The effect of 4-HPR on migration and invasion of EJ cells upon exposure (48 h) to micromole concentrations of 4-HPR was then examined. All the concentrations tested inhibited cell migration and invasion, particularly when cells were treated with 4-HPR at $10 \mu \mathrm{M}$ (Fig. 1B and C).

4-HPR inhibits the migration and invasion of EJ cells by increasing the expression of Wnt5a. Previous studies indicate 
A

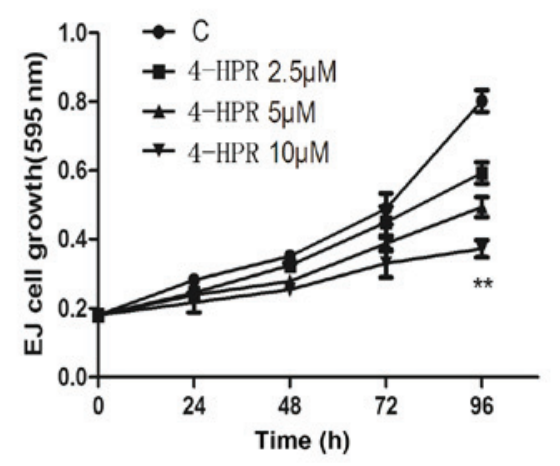

B
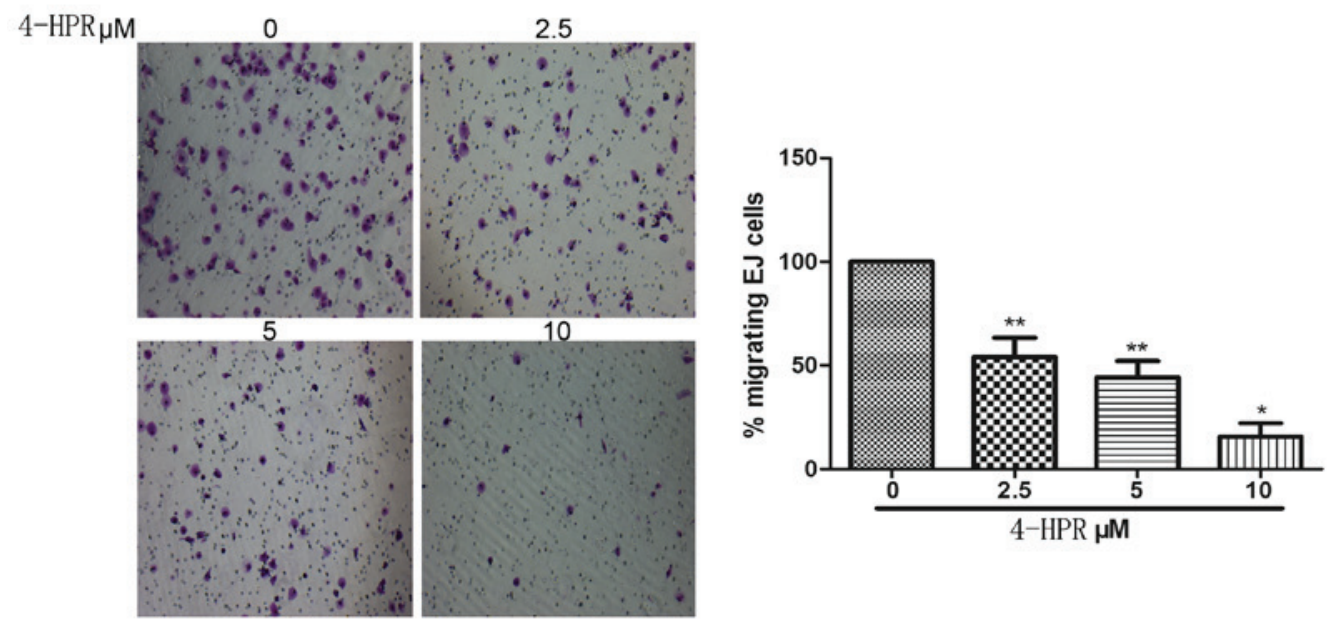

C
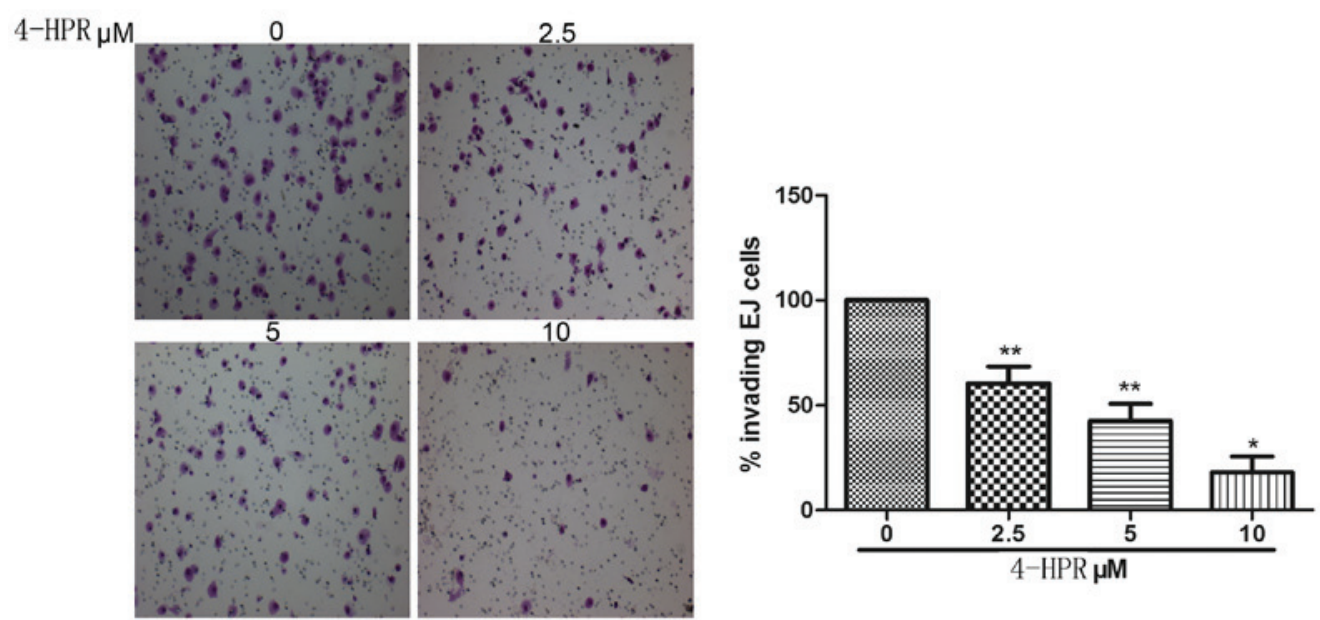

Figure 1. Bladder cancer cell growth, migration and invasion were inhibited by increasing doses of 4-HPR. (A) Cell proliferation, as evaluated by 3-(4,5-dimethylthiazol-2-yl)-2,5-diphenyltetrazolium bromide assay, was significantly inhibited at all the 4-HPR concentrations tested after 96-h exposure. (B) Cell motility through uncoated filters was measured $24 \mathrm{~h}$ after plating in the absence or presence of the indicated concentrations of $4-\mathrm{HPR}$ for $48 \mathrm{~h}$. Then, cells in the lower chamber were stained with crystal violet and photographed with a light microscope (magnification, x100), followed by calculation of the percentage of migrating cells. (C) Invasion assays adopted the same method than migration assays, with the exception of the use of coated filters for $48 \mathrm{~h}$. Data were expressed as the mean \pm standard deviation from three independent experiments. ${ }^{*} \mathrm{P}<0.05,{ }^{* *} \mathrm{P}<0.01$ vs. control. $\mathrm{C}$, control; 4-HPR, $\mathrm{N}$-(4-hydroxyphenyl) retinamide.

that Wnt5a inhibits cell growth, migration and invasiveness of thyroid and colorectal cancer cells $(21,22)$, indicating that Wnt5a may act as a tumor suppressor. Accordingly, the present study examined whether or not expression of Wnt5a can be induced following 4-HPR stimulation. As shown in Fig. 2A, treatment of EJ cells with 4-HPR for $48 \mathrm{~h}$ upregulated the expression of Wnt5a in a dose-dependent manner, as detected by western blot analysis of extracts from EJ cells.
To analyze the efficiency of gene knockdown of Wnt5a, siRNAs were used to knockdown Wnt5a expression in EJ cells, followed by western blot assays. siRNA against human Wnt5a knocked down Wnt5a expression by $>60 \%$, as assessed by immunoblotting in EJ cells (Fig. 2B). To confirm whether Wnt5a activity is involved in the 4-HPR-induced migration and invasion of EJ cells, EJ cells were pre-treated with $5 \mu \mathrm{M}$ 4-HPR for $48 \mathrm{~h}$, and then transfected with control siRNA or Wnt5a siRNA. 


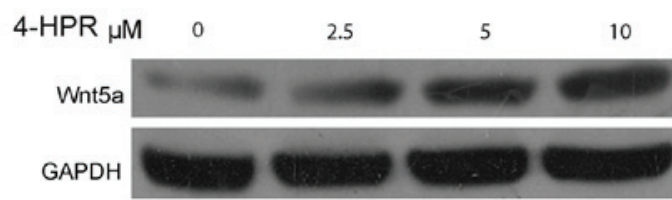

C

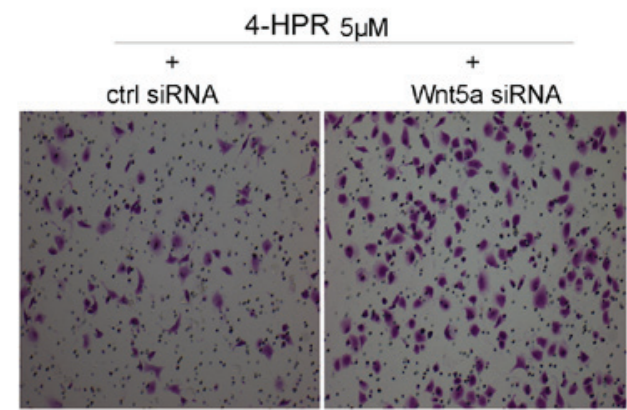

D

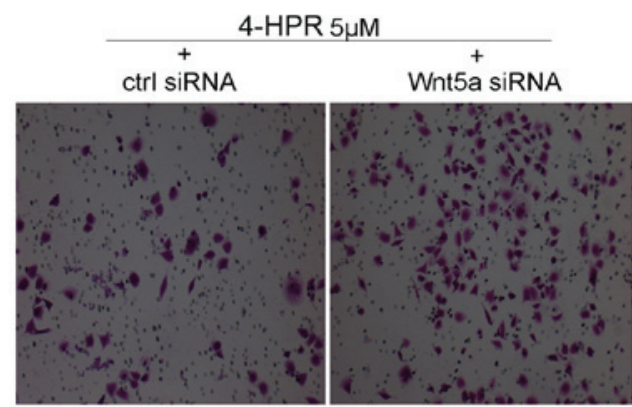

$\mathbf{E}$

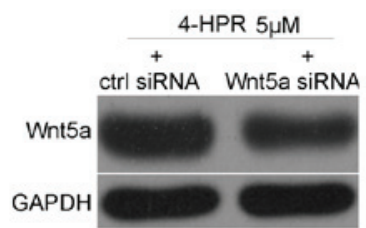

B
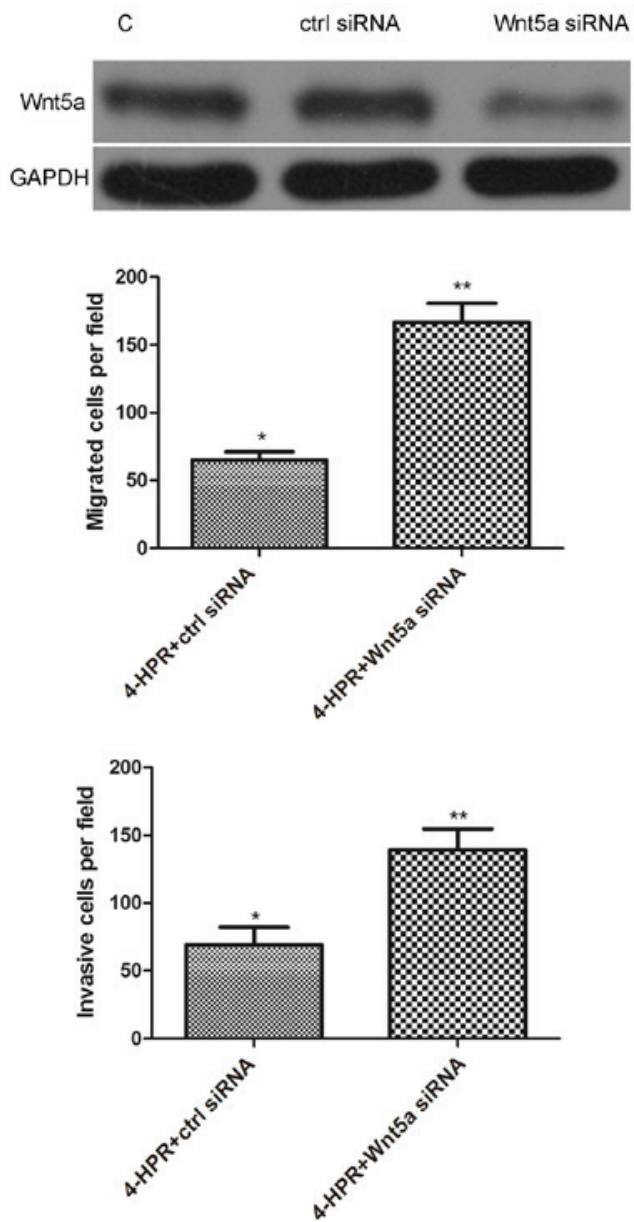

Figure 2. 4-HPR inhibits the migration and invasion of EJ cells by increasing the expression of Wnt5a. (A) EJ cells were incubated with the indicated concentrations of 4-HPR for $48 \mathrm{~h}$, and the protein levels of Wnt5a were measured by western blotting. GAPDH was used as an internal control. (B) EJ cells were transfected with ctrl siRNA and Wnt5a siRNA, and the protein levels of Wnt5a were measured by western blotting. GAPDH was used as an internal control. (C and D) EJ cells were transfected with ctrl siRNA and Wnt5a siRNA for $24 \mathrm{~h}$. After incubation with 4-HPR for $48 \mathrm{~h}$, the cells were analyzed by (C) migration and (D) invasion assays, as described in Fig. 1B and C (magnification, x100). (E) EJ cells were transfected with ctrl siRNA and Wnt5a siRNA for $24 \mathrm{~h}$. After incubation with 4-HPR for $48 \mathrm{~h}$, the protein levels of Wnt5a were measured by western blotting. GAPDH was used as an internal control. Data were expressed as the mean \pm standard deviation from three independent experiments. ${ }^{*} \mathrm{P}<0.05,{ }^{* *} \mathrm{P}<0.01$ vs. control. 4-HPR, N-(4-hydroxyphenyl) retinamide; siRNA, small interfering RNA; Wnt5a, wingless-type mouse mammary tumor virus integration site family, member 5a; GAPDH, glyceraldehyde 3-phosphate dehydrogenase; C, control; ctrl, control.

As shown in Fig. 2C-E, knocking down Wnt5a expression with Wnt5a siRNA could inhibit the migration and invasion of EJ cells, and decrease the expression of Wnt5a, compared with control siRNA transfection. Collectively, these data support the idea that 4-HPR inhibits the migration and invasion of bladder cancer cells through high expression of Wnt5a.

4-HPR inhibits the migration and invasion of EJ cells by stimulating Wnt5a activity and causing the phosphorylation of JNK on Thr183/Tyr185. As shown in Fig. 3A, treatment of EJ cells with 4-HPR for $48 \mathrm{~h}$ upregulated the phosphorylation of JNK in a dose-dependent manner, as detected by western blot analysis of extracts from EJ cells. To further confirm whether the phosphorylation of JNK could be induced following Wnt5a stimulation, EJ cells were exposed to 4-HPR for $48 \mathrm{~h}$ to stimulate the activity of Wnt5a, and the phosphorylation of JNK was induced upon stimulation with 4-HPR, as assessed by western blot analysis. In addition, the phosphorylation of JNK was enhanced by 4-HPR, and this 4-HPR-induced enhancement was abrogated by Wnt5a siRNA (Fig. 3B). Additionally, knocking down Wnt5a expression abrogated the 4-HPR-induced migration and invasion of EJ cells, further demonstrating the critical roles of phosphorylation of JNK, induced by Wnt5a signaling, in the migration and invasion of EJ cells (Fig. 3C and D).

Next, the effect of phosphorylation of JNK on the migration and invasion of EJ cells was examined. For this purpose, EJ cells were pre-treated with the JNK inhibitor SP600125 for $30 \mathrm{~min}$, and then exposed to 4-HPR for $48 \mathrm{~h}$. The migration and invasion of EJ cells were inhibited by 4-HPR, and this 4-HPR-induced inhibition was abrogated by the presence of the JNK inhibitor SP600125, while the 4-HPR-induced phosphorylation of JNK was also abrogated by SP600125 (Fig. 3E). These data indicate that 4-HPR stimulates the phosphorylation and subsequent activity of JNK via the Wnt5a signaling pathway, leading to the inhibition of migration and invasion in EJ bladder cancer cells. 

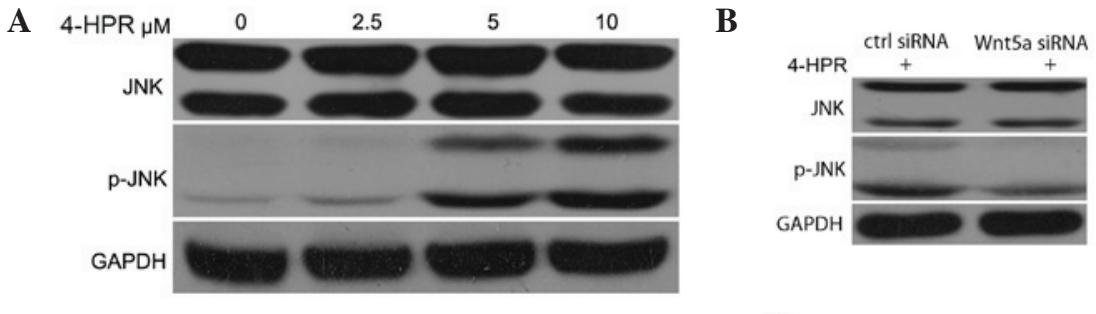

C
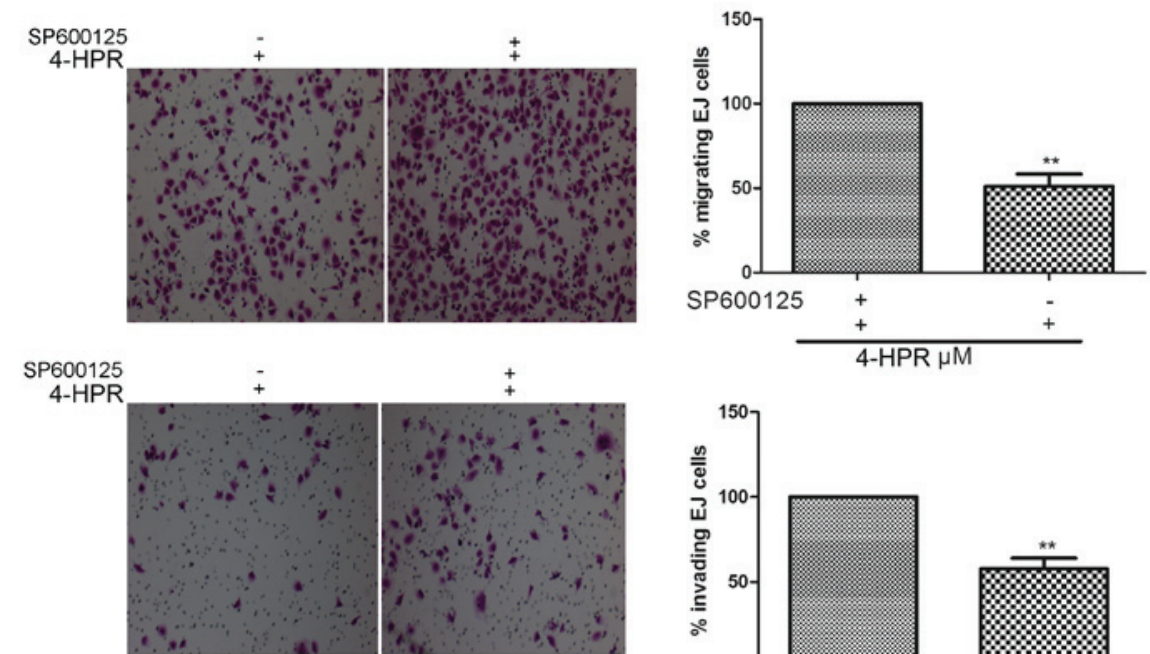

$\mathbf{E}$
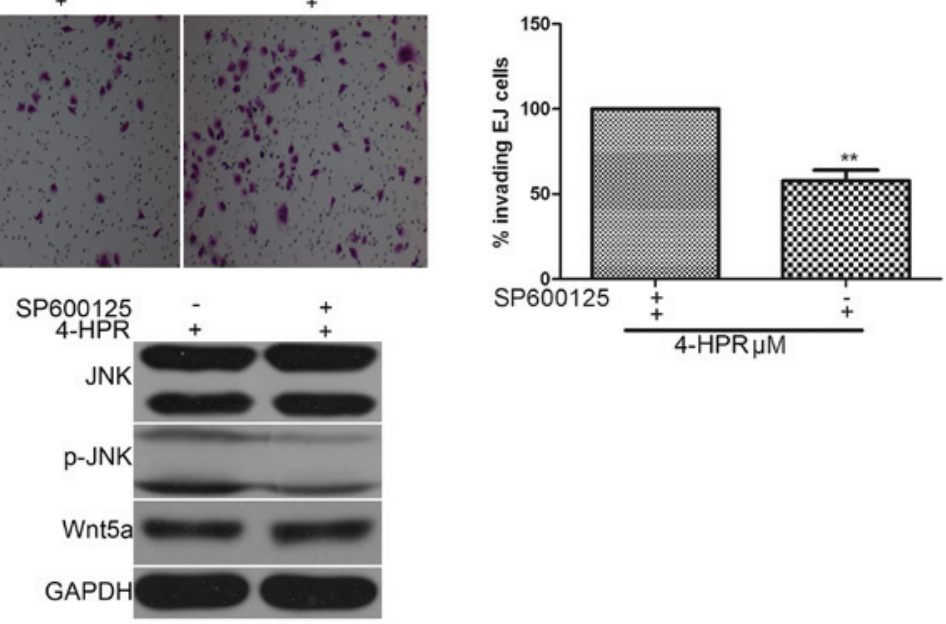

Figure 3. 4-HPR inhibits the migration and invasion of EJ cells by stimulating Wnt5a activity and JNK phosphorylation. (A) EJ cells were incubated with the indicated concentrations of 4-HPR for $48 \mathrm{~h}$, and the protein levels of JNK and pJNK were measured by western blotting. GAPDH was used as an internal control. (B) EJ cells were treated with $5 \mu \mathrm{M} 4$-HPR for $48 \mathrm{~h}$, and then transfected with control or Wnt5a small interfering RNA. Cell lysates were assayed for JNK and pJNK by western blotting with anti-JNK, anti-pJNK and anti-GAPDH antibodies. (C-E) EJ cells were treated with SP600125, an inhibitor of JNK, and then incubated with $5 \mu \mathrm{M}$ 4-HPR for $48 \mathrm{~h}$. Cell migration and invasion were measured by (C) migration and (D) invasion assays (magnification, x100), (E) and the protein extracts were analyzed by western blotting for JNK and pJNK. Data were expressed as the mean \pm standard deviation from three independent experiments. ${ }^{*} \mathrm{P}<0.05,{ }^{* *} \mathrm{P}<0.01$ vs. control. 4-HPR, $\mathrm{N}$-(4-hydroxyphenyl) retinamide; siRNA, small interfering RNA; Wnt5a, wingless-type mouse mammary tumor virus integration site family, member 5a; GAPDH, glyceraldehyde 3-phosphate dehydrogenase; ctrl, control; JNK, c-Jun N-terminal kinase; pJNK, phosphorylated c-Jun N-terminal kinase.

A

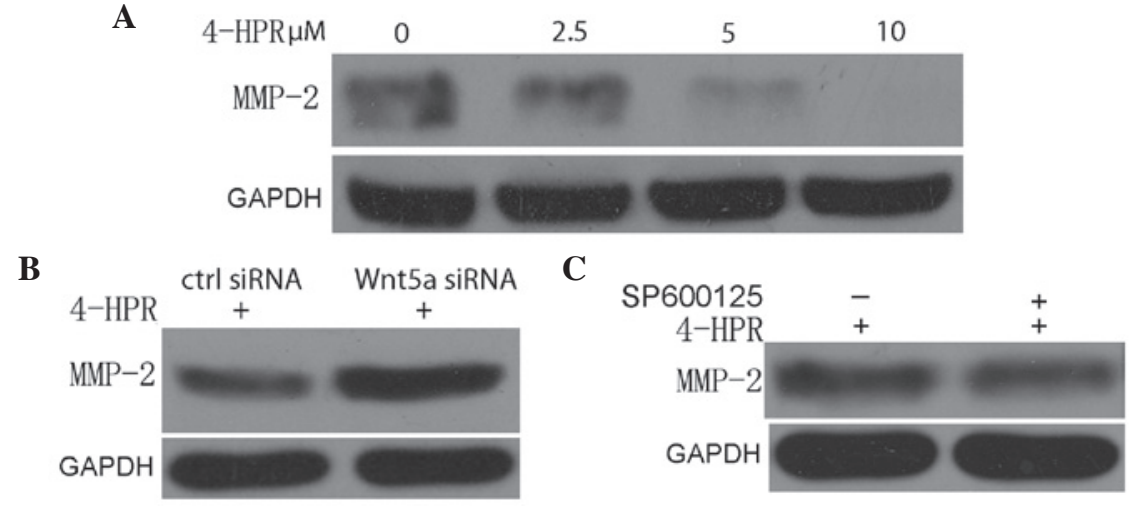

2.5

5 10

Figure 4. 4-HPR inhibits the migration and invasion of bladder cancer cells through suppression of MMP-2. (A) EJ cells were incubated with the indicated concentrations of 4-HPR for $48 \mathrm{~h}$, and the protein levels of MMP-2 were measured by western blotting. GAPDH was used as an internal control. (B) EJ cells were treated with $5 \mu \mathrm{M} 4$-HPR for $48 \mathrm{~h}$, and then transfected with control or Wnt5a small interfering RNA. Cell lysates were assayed for MMP-2 by western blotting with an anti-MMP-2 antibody. (C) EJ cells were treated with SP600125, an inhibitor of c-Jun N-terminal kinase, and then incubated with $5 \mu$ M 4-HPR for $48 \mathrm{~h}$. Next, the protein extracts were analyzed by western blotting for MMP-2. 4-HPR, N-(4-hydroxyphenyl) retinamide; siRNA, small interfering RNA; Wnt5a, wingless-type mouse mammary tumor virus integration site family, member 5a; GAPDH, glyceraldehyde 3-phosphate dehydrogenase; ctrl, control; MMP-2, matrix metalloproteinase-2. 
4-HPR inhibits the migration and invasion of bladder cancer cells through the suppression of MMP-2. The evidence for MMPs as active contributors to cancer progression comes from previous animal studies (23). In previous transplantation assays, relatively benign cancer cells acquired malignant properties when MMP expression was upregulated (23). Conversely, highly malignant cells became less aggressive when MMP expression or activity was reduced (12). It is well established that MMP-2 is involved in bladder cancer migration and invasion. In the present study, 4-HPR decreased the expression of MMP-2 in a dose-dependent manner, when the migration and invasion of EJ cells were inhibited (Fig. 4A). Furthermore, this suppressed expression of MMP-2 was abrogated by a suppressed expression of Wnt5a, compared with control siRNA transfection (Fig. 4B). These results indicate that 4-HPR inhibits the migration and invasion of EJ cells through inhibiting MMP-2 expression, which is regulated by Wnt5a signaling.

The present study demonstrated that 4-HPR inhibited the migration and invasion of EJ cells through the suppression of MMP-2, which was regulated by Wnt5a signaling, although the suppressed phosphorylation of JNK did not appear to have a direct effect on the expression of MMP-2, as assessed by western blot analysis.

\section{Discussion}

4-HPR has potent chemo-preventive and anti-metastatic effects in several animal models (24-26), and is currently under investigation on clinical trials for use as a preventive agent for ovarian carcinoma, lung carcinoma and breast neoplasia $(27,28)$. In the present study, 4-HPR inhibited the migration and invasion of EJ cells in a dose-dependent manner. MMPs were prime candidates for invasion and metastasis, since MMP family members collectively degrade all the structural components of the ECM (29). In addition, MMPs are upregulated in virtually all human and animal tumors, as well as in the majority of tumor cell lines (30). Previous studies indicate that 4-HPR inhibits breast cancer cell invasion through inhibiting MMP-9 expression (31). The present results indicate that 4-HPR inhibits the expression of MMP-2, suggesting that 4-HPR can inhibit cell migration and invasion by suppressing MMP-2 expression.

Wnt5a, as one of the most highly investigated non-canonical Wnts, has been implicated in almost all aspects of cancer development (32). Wnt5a plays essential roles in developmental and physiological processes by regulating several cell functions, including proliferation, differentiation, apoptosis, survival, adhesion, migration and polarity (21). A primary observation of the present study is that 4-HPR inhibits the migration and invasion of EJ bladder cancer cells, which was fully abolished by Wnt5a siRNA, confirming that 4-HPR inhibits the migration and invasion of EJ bladder cancer cells via stimulation of Wnt5a. In addition, it has already been reported that mouse Wnt5a is capable of activating JNK in cultured cells (33). The present findings suggest that 4-HPR stimulates the phosphorylation of JNK and its activity via the Wnt5a signaling pathway, leading to inhibited migration and invasion of EJ bladder cancer cells. In addition, the present data support the idea that 4-HPR inhibits MMP-2 activity via the Wnt5a signaling pathway, leading to inhibited migration and invasion of EJ bladder cancer cells.

In conclusion, the present study reports that 4-HPR stimulates the activation of Wnt5a in bladder cancer, and demonstrates that 4-HPR inhibits the migration and invasion of bladder cancer cells though stimulating Wnt5a activity, causing the downregulation of MMP-2 expression and enhancing enhancement JNK phosphorylation. Thus, 4-HPR may be a potent anti-invasive and anti-metastatic agent that works via the Wnt5a/JNK and Wnt5a/MMP-2 signaling pathways in bladder cancer cells. Therefore, 4-HPR is a potential chemotherapeutic candidate with a broad spectrum of anticancer activities. Despite these findings, further studies on the mechanism of Wnt5a/JNK and Wnt5a/MMP-2 signaling, as well as future experiments using in vivo models, are required.

\section{Acknowledgements}

The present study was supported by a grant from the National Natural Science Foundation of China (Beijing, China; grant no. 303162520).

\section{References}

1. Siegel R, Naishadham D and Jemal A: Cancer statistics, 2012. CA Cancer J Clin 62: 10-29, 2012.

2. Sievert KD, Amend B, Nagele U, Schilling D, Bedke J, Horstmann M, Hennenlotter J, Kruck S and Stenzl A: Economic aspects of bladder cancer: What are the benefits and costs? World J Urol 27: 295-300, 2009.

3. Babjuk M, Oosterlinck W, Sylvester R, Kaasinen E, Böhle A, Palou-Redorta J and Rouprêt M; European Association of Urology (EAU): EAU guidelines on non-muscle-invasive urothelial carcinoma of the bladder, the 2011 update. Eur Urol 59: 997-1008, 2011.

4. Dowlatshahi K, Mehta RG, Thomas CF, Dinger NM and Moon RC: Therapeutic effect of N-(4-hydroxyphenyl) retinamide on $\mathrm{N}$-methyl-N-nitrosourea-induced rat mammary cancer. Cancer Lett 47: 187-192, 1989.

5. Formelli F and Cleris L: Synthetic retinoid fenretinide is effective against a human ovarian carcinoma xenograft and potentiates cisplatin activity. Cancer Res 53: 5374-5376, 1993.

6. Pollard M and Luckert PH: The inhibitory effect of 4-hydroxyphenyl retinamide (4-HPR) on metastasis of prostate adenocarcinoma-III cells in Lobund-Wistar rats. Cancer Lett 59: 159-163, 1991.

7. Kurie JM1, Lee JS, Khuri FR, Mao L, Morice RC, Lee JJ, Walsh GL, Broxson A, Lippman SM, Ro JY, et al: $\mathrm{N}$-(4-hydroxyphenyl)retinamide in the chemoprevention of squamous metaplasia and dysplasia of the bronchial epithelium. Clin Cancer Res 6: 2973-2979, 2000.

8. Costa A, Formelli F, Chiesa F, Decensi A, De Palo G and Veronesi U: Prospects of chemoprevention of human cancers with the synthetic retinoid fenretinide. Cancer Res 54 (7 Suppl): 2032s-2037s, 1994.

9. Stamenkovic I: Matrix metalloproteinases in tumor invasion and metastasis. Semin Cancer Biol 10: 415-433, 2000.

10. Basset P, Okada A, Chenard MP, Kannan R, Stoll I, Anglard P, Bellocq JP and Rio MC: Matrix metalloproteinases as stromal effectors of human carcinoma progression: Therapeutic implications. Matrix Biol 15: 535-541, 1997.

11. Johnsen M, Lund LR, Rømer J, Almholt K and Danø K: Cancer invasion and tissue remodeling: Common themes in proteolytic matrix degradation. Curr Opin Cell Biol 10: 667-671, 1998.

12. Egeblad M and Werb Z: New functions for the matrix metalloproteinases in cancer progression. Nat Rev Cancer 2: 161-174, 2002.

13. Srivastava P, Kapoor R and Mittal RD: Association of single nucleotide polymorphisms in promoter of matrix metalloproteinase-2, 8 genes with bladder cancer risk in Northern India. Urol Oncol 31: 247-254, 2013. 
14. Shan Y, Zhang L, Bao Y, Li B, He C, Gao M, Feng X, Xu W, Zhang $X$ and Wang S: Epithelial-mesenchymal transition, a novel target of sulforaphane via COX-2/MMP2, 9/Snail, ZEB1 and miR-200c/ZEB1 pathways in human bladder cancer cells. J Nutr Biochem 24: 1062-1069, 2013.

15. Logan CY and Nusse R: The Wnt signaling pathway in development and disease. Annu Rev Cell Dev Biol 20: 781-810, 2004.

16. Wong GT, Gavin BJ and McMahon AP: Differential transformation of mammary epithelial cells by Wnt genes. Mol Cell Biol 14: 6278-6286, 1994.

17. Nishita $M$, Enomoto $M$, Yamagata $K$ and Minami $Y$ : Cell/tissue-tropic functions of Wnt5a signaling in normal and cancer cells. Trends Cell Biol 20: 346-354, 2010.

18. Oishi I, Suzuki H, Onishi N, Takada R, Kani S, Ohkawara B, Koshida I, Suzuki K, Yamada G, Schwabe GC, et al: The receptor tyrosine kinase Ror2 is involved in non-canonical Wnt5a/JNK signaling pathway. Genes Cells 8: 645-654, 2003.

19. Huang C, Rajfur Z, Borchers C, Schaller MD and Jacobson K: JNK phosphorylates paxillin and regulates cell migration. Nature 424: 219-223, 2003.

20. Kikuchi A, Yamamoto H, Sato A and Matsumoto S: Wnt5a: Its signaling, functions and implication in diseases. Acta Physiol (Oxf) 204: 17-33, 2012.

21. Dejmek J, Dejmek A, Säfholm A, Sjölander A and Andersson T: Wnt-5a protein expression in primary dukes B colon cancers identifies a subgroup of patients with good prognosis. Cancer Res 65: 9142-9146, 2005.

22. Kremenevskaja N, von Wasielewski R, Rao AS, Schöfl C, Andersson T and Brabant G: Wnt-5a has tumor suppressor activity in thyroid carcinoma. Oncogene 24: 2144-2154, 2005.

23. Coussens LM and Werb Z: Matrix metalloproteinases and the development of cancer. Chem Biol 3: 895-904, 1996.
24. Green A, Shilkaitis A and Christov K: 4-(hydroxyphenyl) retinamide selectively inhibits the development and progression of ductal hyperplastic lesions and carcinoma in situ in mammary gland. Carcinogenesis 20: 1535-1540, 1999.

25. Shaker MR, Yang G, Timme TL, Park SH, Kadmon D, Ren C, Ji X, Lee HM, Sehgal I, Anzano M, et al: Dietary 4-HPR suppresses the development of bone metastasis in vivo in a mouse model of prostate cancer progression. Clin Exp Metastasis 18: 429-438, 2000.

26. Hursting SD, Perkins SN, Phang JM and Barrett JC: Diet and cancer prevention studies in p53-deficient mice. J Nutr 131 (11 Suppl): 3092S-3094S, 2001.

27. Cai J and Jones DP: Superoxide in apoptosis. Mitochondrial generation triggered by cytochrome c loss. J Biol Chem 273: 11401-11404, 1998.

28. Levi MS, Borne RF and Williamson JS: A review of cancer chemopreventive agents. Curr Med Chem 8: 1349-1362, 2001.

29. McCawley LJ and Matrisian LM: Matrix metalloproteinases: multifunctional contributors to tumor progression. Mol Med Today 6: 149-156, 2000.

30. Coussens LM, Fingleton B and Matrisian LM: Matrix metalloproteinase inhibitors and cancer: Trials and tribulations. Science 295: 2387-2392, 2002.

31. Kang H, Lee M, Choi KC, Shin DM, Ko J and Jang SW: $\mathrm{N}$-(4-hydroxyphenyl) retinamide inhibits breast cancer cell invasion through suppressing NF-KB activation and inhibiting matrix metalloproteinase-9 expression. J Cell Biochem 113: 2845-2855, 2012.

32. Nusse R: Wnt signaling in disease and in development. Cell Res 15: 28-32, 2005.

33. Yamanaka H, Moriguchi T, Masuyama N, Kusakabe M, Hanafusa H, Takada R, Takada S and Nishida E: JNK functions in the non-canonical Wnt pathway to regulate convergent extension movements in vertebrates. EMBO Rep 3: 69-75, 2002. 